\title{
Primeiro relato de Lutzomyia cortelezzii (Brèthes, 1923) na Cidade de Ilhéus, BA
}

\author{
First report of Lutzomyia cortelezzii ( Brèthes, 1923) in Ilhéus City, Bahia
}

\author{
Sílvia Maria Santos Carvalho ${ }^{1}$, Eunice Matos Guimarães ${ }^{1}$, Yasmine Barbosa de Souza ${ }^{1}$, \\ Vania Bastos Braga ${ }^{1}$, Letícia Carvalho Vianna ${ }^{2}$, Paulo Raimundo Barbosa dos Santos ${ }^{3}$, \\ Sebastião de Souza ${ }^{3}$, Reinaldo Silva ${ }^{3}$, Adevegistro Pereira ${ }^{3}$ e Missias da Hora Leite ${ }^{3}$
}

\begin{abstract}
RESUMO
A Lutzomyia cortelezzii foi encontrada na área urbana da Cidade de Ilhéus, Bahia, Brasil. A aparição deste flebotomíneo em $100 \%$ das $288 \mathrm{~h}$ de capturas no território ilheense é fato inédito. As coletas foram realizadas através de armadilhas luminosas tipo CDC, de dezembro/2003 a maio/2004, num total de 81 exemplares coletados.
\end{abstract}

Palavras-chaves: Lutzomyia cortelezzii. Flebotomíneo. CDC. Ilhéus.

\begin{abstract}
Lutzomyia cortelezzii was found in the urban area of Ilhéus, Bahia, Brazil. The appearance this phlebotomine in 100\% of 288 hours of capture in Ilhéus' territory is an unpublished fact. The collection was by CDC light traps between December 2003 and May 2004, with a total of 81 collected samples.
\end{abstract}

Key-words: Lutzomyia cortelezzii. Phlebotomine. CDC. Ilhéus.

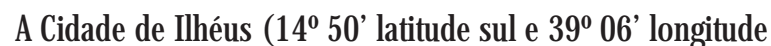
oeste), localizada ao sul do litoral baiano, a $462 \mathrm{~km}$ da capital do estado, com área de clima tropical quente e alta pluviosidade anual (1500 - 2000mm), com remanescentes de Mata Atlântica e grande extensão de plantações de cacau ${ }^{4}$, é endêmica para leishmaniose tegumentar e já registrou a presença de espécies de flebotomíneos como Lutzomyia intermedia, L whitmani, L fischeri e L migonei na zona rural ${ }^{1}$, sem relatos na área urbana onde, de fato, não há registros de estudos entomológicos. Em face disso, realizamos capturas de flebotomíneos em sete diferentes bairros desta cidade ( ao norte, Novo Ilhéus e Iguape; a oeste, Teotônio Vilela; ao sul, Nossa Senhora da Vitória; e bairros centrais, Conquista, Princesa Isabel e Malhado), na tentativa de registrar 0 achado de espécies.

Os exemplares foram capturados com uso de armadilhas luminosas tipo CDC (Center on Disease Control) ${ }^{6}$, armadas às 18:00h e retiradas às 06:00h do dia seguinte, a 1,5m de altura do solo, entre os meses de dezembro/2003 e maio/2004, uma semana por mês, cada dia dedicado a um ou dois bairros, com umidade relativa do ar variando entre $58 \%$ e $83 \%$, e a temperatura na faixa entre $22^{\circ} \mathrm{C}$ e $36^{\circ} \mathrm{C}$, em quintais de casas com algum tipo de árvore frutífera e/ou abrigos de animais existentes, totalizando $288 \mathrm{~h}$ de trabalho, com 81 exemplares capturados ( 43 fêmeas e 38 machos), pertencentes à espécie L cortelezzii, presente em todos os bairros, à exceção de Nossa Senhora da Vitória e Novo Ilhéus. Os exemplares foram devidamente armazenados em álcool 70\%, clarificados em KOH $10 \%$ e montados em Berlese $e^{8}$. Vale mencionar que esta espécie é usada por alguns pesquisadores como sinonímia de L sallesi, constituindo, junto à Lutzomyia corumbaensis, o complexo L cortelezzii ${ }^{3}$.

0 achado de L cortelezzii em buracos de árvores na Bahia já foi descrito² ${ }^{2}$ porém a sua aparição na área urbana de Ilhéus é fato inédito, sendo o único flebotomíneo presente. Ao contrário, em trabalho realizado na área urbana de Maringá, $\mathrm{PR}^{7}$, percebeuse que estes insetos responderam por apenas $0.03 \%$ do total

\footnotetext{
1. Departamento de Ciências Biológicas da Universidade Estadual de Santa Cruz, Ilhéus, BA. 2. Instituto de Ciências Biológicas da Universidade Católica do Salvador,

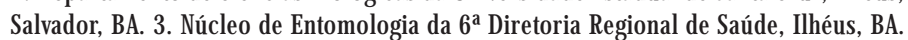

Trabalho financiado pela UESC (Universidade Estadual de Santa Cruz), FAPESB (Fundação de Amparo à Pesquisa do Estado da Bahia) e 6a DIRES (Diretoria Regional de Saúde).

Endereço para correspondência: Dra. Silvia Maria Santos Carvalho. Rodovia Ilhéus Itabuna, Km 16, 45.650-000 Ilhéus, BA.

Tel: 5573 3680-5268, Fax: 5573 3680-5226.

e-mail: sissa@uesc.br - sissacarvalho@yahoo.com

Recebido para publicação em 7/6/2004

Aceito em 6/6/2005
} 
registrado. Já em estudo também realizado em área urbana, entre os meses de fevereiro de 1999 e fevereiro de 2000, na Cidade de Corumbá, foi notificada a presença de L cortelezzii entre outras 27 espécies capturadas, respondendo por 3,4\% do achado, com 42 exemplares coletados ${ }^{5}$.

Em Ilhéus, 0 inseto foi observado com maior freqüência nos bairros centrais, onde, apesar da infra-instrutura urbana, há casas com quintais, com presença de vegetação, em geral árvores frutíferas e, não raramente, abrigos de animais como cães e galinhas. Entretanto, nos bairros periféricos, onde são observadas áreas de transição rural-urbana, a freqüência dos insetos foi baixa ou nula.

A presença de L cortelezzii na zona urbana de Ilhéus não quer dizer que este flebotomíneo responda pela capacidade vetorial nesta área, especialmente porque a densidade é relativamente baixa. Adicionalmente, os casos humanos, verdadeiramente positivos de leishmaniose ali registrados, muito provavelmente são oriundos da zona rural. Além disso, no Brasil não há relatos de incriminação desta espécie na transmissão da doença. Ao contrário, no norte da Argentina, há a suspeita de que a mesma seja responsável pelo aparecimento de casos humanos, em função de a sua presença coincidir com a faixa de distribuição da doença neste país e, sobretudo, por ter sido observada freqüentando ambiente domiciliar, alimentando-se de sangue humano e pela associação com animais silvestres².

Baseado no exposto, é razoável pensar que é pouco provável o estabelecimento de um foco de transmissão na área urbana ilheense, especialmente devido à ausência de insetos reconhecidamente vetores em potencial. Entretanto, há registros de casos como sendo autóctones desta área. Apesar disso, vale mencionar que as fichas de registros não contêm informações suficientes para realização de um levantamento sobre a real origem dos casos. Este é apenas um dos fatores que vem sendo estudados no levantamento epidemiológico, complementar a este trabalho.

\section{AGRADECIMENTOS}

Às pessoas que permitiram 0 acesso às suas residências para que as armadilhas fossem montadas.

\section{REFERÊNCIAS BIBLIOGRÁFICAS}

1. Azevedo ACR, Vilela ML, Souza NA, Andrade-Coelho CA, Barbosa FA, Firmo ALS, Rangel EF. The sand fly fauna (Diptera: Psychodidade: Phebotominae) of a focus cutaneous leishmaniasis in Ilhéus, State of Bahia, Brazil. Memórias do Instituto Oswaldo Cruz 91:75-79, 1996.

2. Forattini OP. Entomologia Médica. Edgard Blucher Ltda, Coedição Editora da Universidade do Estado de São Paulo, 4ํvolume, 1973.

3. Galati EAB, Nunes, VLB, Oshiro, ET, Rego-Jr, FA. Nova espécie de Phlebotominae, Lutzomyia corumbanesis, sp. N. (Diptera, Psychodidae) do complexo Lutzomyia cortelezzii. Revista Brasileira de Entomologia 33: 465-475, 1989.

4. http://www.ecobahia.com.br/m-ios-itb-ilheus.htm Disponível em: 02/05/ 2004.

5. Oliveira AG, A Filho JD, Falcão AL, Brazil RP. Estudo de flebotomíneos ( Diptera, Psychodidae,Phlebotominae) na zona urbana da Cidade de Campo Grande, Mato Grosso do Sul, Brasil,1999-2000. Caderno de Saúde Pública 19 (4) , 2003.

6. Sudia WD, Chamberlain RW. Battery-operated light trap, an improved model. Mosquito News 22: 126-129, 1962.

7. Teodoro U, Ruhl JB, Rodríguez M, Santos ES, Santos DR, Maróstica LMF. Flebotomínos coletados em matas silvestres de zoológico no perímetro urbano de Maringá, Sul do Brasil. Estudo Preliminar. Revista da Sociedade Brasileira de Medicina Tropical 31: 517-522, 1998.

8. Young DG, Ducan MA. Guide to the identification and geographic distribution of Lutzomyia Sand Flies in Mexico, the West Indies, Central and South American (Diptera: Psychodidae). Memories of the American Entomological Institute 54: 1-881, 1994. 